Elsevier Editorial System(tm) for European Journal of Internal Medicine Manuscript Draft

Manuscript Number: EJIM-D-14-00203R1

Title: Non-alcoholic fatty liver disease, metabolic syndrome and patatin-like phospholipase domaincontaining protein3 (PNPLA3) gene variants

Article Type: Original Article

Keywords: Non-alcoholic fatty liver

Non-alcoholic steatohepatitis

Metabolic syndrome

PNPLA3 polymorphism

Corresponding Author: Prof Francesco Angelico, MD

Corresponding Author's Institution: University La Sapienza

First Author: Maria Del Ben

Order of Authors: Maria Del Ben; Licia Polimeni; Monica Brancorsini; Alessia Di Costanzo; Laura D'Erasmo; Francesco Baratta; Lorenzo Loffredo; Daniele Pastori; Pasquale Pignatelli; Francesco Violi; Marcello Arca; Francesco Angelico, MD

Abstract: Background \& Aims: Non-alcoholic fatty liver disease was traditionally interpreted as a condition which may progress to liver-related complications. However, the increased mortality is primarily a result of cardiovascular diseases. It has been suggested that fatty liver can be considered as the hepatic consequence of the metabolic syndrome. The aim was to describe the different clinical presentations of non-alcoholic fatty liver disease on the basis of the patatin-like phospholipase domain-containing protein3 (PNPLA3) rs738409 gene variant.

Methods Fatty liver was defined by ultasonographic Hamaguchi's criteria in 211 consecutive subjects with non-alcoholic fatty liver disease. The rs738409 polymorphism was determined by TaqMan assays. Metabolic syndrome was defined according to ATPIII modified criteria. Results Prevalence of PNPLA3-148II, PNPLA3-148IM, and PNPLA3-148MM genotypes was 45.0\%, $40.7 \%$, and $14.3 \%$ respectively. Prevalence of metabolic syndrome progressively increased with the severity of liver steatosis (from $52.5 \%$ to $65.2 \%$, and $82.3 \%$ respectively, $\mathrm{p}<0.01$ ). The PNPLA3$148 \mathrm{MM}$ group had significantly lower mean serum triglycerides $(\mathrm{p}<0.001)$, Framingham cardiovascular risk score $(\mathrm{p}<0.01)$ and lower prevalence of metabolic syndrome $(\mathrm{p}<0.05)$ and its components. Age and HOMA-IR were positive independent predictors of metabolic syndrome, while a negative independent association was found between metabolic syndrome and the homozygotes PNPLA3 I148M variant.

Conclusions We suggest a lower prevalence of MetS and reduced cardiovascular risk in NAFLD patients with PNPLA3MM genotype. 

To the Editor,

European Journal of Internal Medicine

Please, find enclosed a carefully revised version of our manuscript "Non-alcoholic fatty liver disease, metabolic syndrome and patatin-like phospholipase domain-containing protein3 (PNPLA3) gene variants" The manuscript has been extensively revised taking in to consideration all the comments, questions and suggestions of the reviewers. A point to point reply to the criticisms of each Reviewer has been prepared. The revised text clearly indicates where changes have been made by using a red font.

Francesco Angelico

$09 / 05 / 2014$ 
We wish to confirm that there are no known conflicts of interest associated with this publication and there has been no significant financial support for this work that could have influenced its outcome.

We confirm that the manuscript has been read and approved by all named authors and that there are no other persons who satisfied the criteria for authorship but are not listed. We further confirm that the order of authors listed in the manuscript has been approved by all of us.

We confirm that we have given due consideration to the protection of intellectual property associated with this work and that there are no impediments to publication, including the timing of publication, with respect to intellectual property. In so doing we confirm that we have followed the regulations of our institutions concerning intellectual property.

We further confirm that any aspect of the work covered in this manuscript that has involved either experimental animals or human patients has been conducted with the ethical approval of all relevant bodies and that such approvals are acknowledged within the manuscript.

We understand that the Corresponding Author is the sole contact for the Editorial process (including Editorial Manager and direct communications with the office). He is responsible for communicating with the other authors about progress, submissions of revisions and final approval of proofs. We confirm that we have provided a current, correct email address which is accessible by the Corresponding Author.

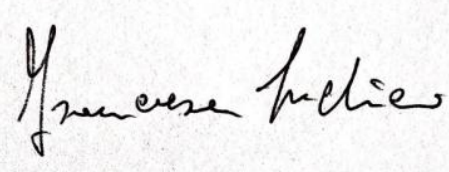

Francesco Angelico

(on behalf of all co-Authors) 
To the Editor,

European Journal of Internal Medicine

Please, find enclosed a carefully revised version of our manuscript "Non-alcoholic fatty liver disease, metabolic syndrome and patatin-like phospholipase domain-containing protein3 (PNPLA3) gene variants" The manuscript has been extensively revised taking in to consideration all the comments, questions and suggestions of the reviewers. A point to point reply to the criticisms of each Reviewer has been prepared.

The revised text clearly indicates where changes have been made by using a red font.

Francesco Angelico

$09 / 05 / 2014$

POINT-TO-POINT REPLY

Reviewers' comments:

Reviewer \#1: The authors evaluated whether PNPLA3 gene variants may predict different clinical phenotypes in Italian patients with US diagnosis of NAFLD. 211 subjects were evaluated. The study is of potential interest, but the author's conclusions deserve caution and could not be full supported by the results. In particular the conclusion: "We demonstrated that NAFLD does not always represent the hepatic manifestation of MetS since PNPLA3-I148M gene variant in our study was not associated with MetS and cardiometabolic risk in individuals with NAFLD", needs some consideration.

a.The major point is that the number of patients in the PNPLA3-I148M group is very low (30 subjects) to draw definitive conclusions based on genetic polymorphism;

REPLY: The rather low number of patients in the PNPLA3-I148M group has been indicated in the Discussion as a possible limitation of the study conclusions.

b. moreover, $50 \%$ of subjects in this group had MetS, thus an association was present, although, $20 \%$ less than other polymorphisms;

REPLY: We agree with the Reviewer that the association with MetS is present. However, it is less than in the other genotypes.

c. lower levels of tryglicerides were associated with the polymorphism IM, thus, this may account for lower prevalence of Mets;

REPLY: the lower levels of triglycerides have been commented in the Discussion.

d. another important point is the significant higher number of female $(50 \%)$ in this group that may have determined the lower cardiometabolic risk, considering the weigh that gender has in the Framingham score.

REPLY: the higher number of females in the MM group has been indicated in the Discussion as a possible limitation in the interpretation of the lower CV risk in this group 
On these bases, the subsequent conclusion:" we suggest that PNPLA3 polymorphisms may help to differentiate clinical phenotypes of NAFLD and be an important candidate gene to discriminate NAFLD with possible metabolic and cardiovascular consequences from fatty liver at risk for liver related complications", cannot be done.

REPLY: The above sentence has been removed. The first sentence of the Discussion and the conclusions in the Abstract and in the main text have been modified following the Reviewer's suggestions.

I believe that the results of this study could be of interest, but the conclusions must be in according to the data and the authors should better underline the above reported limitation of the study. The authors could suggest the possible lower prevalence of MetS and CV risk to be evaluated in "ad hoc" future studies.

REPLY: The limitations of the study have been further highlighted. All Reviewer's suggestions have been taken into consideration and the relevant changes have been made (see, beginning of the Discussion, limitations of the study and the Conclusions).

It is also obvious that the first sentence of "Discussion": "Our results show for the first time that PNPLA3I148M gene variant is not associated with MetS and cardiometabolic risk in a large series of individuals with NAFLD", must be deleted.

REPLY: The first sentence of the Discussion has been deleted.

A further limitation of this study is that NAFLD diagnosis has been done by US. Thus, a significant number of subjects with steatosis less than $30 \%$ have been missed; moreover the differentiation in mild, moderate or severe is highly operator dependent and approximate.

REPLY: NAFLD diagnosis done by US is already reported as a possible limitation of the study in the Discussion. We acknowledge that Hamaguchi score does not specifically predict NASH and that the differentiation in mild, moderate and severe steatosis is highly operator dependent and approximate. However, in our series, groups of patients with different steatosis severity were well characterized (see table 1) and all US evaluations were performed by the same well skilled operator.

Reviewer \#2: Minor points. Initial results: data on steatosis are reported as \% but they are absolute numbers.

REPLY: The reviewer is right. Data on steatosis are absolute numbers.

Too much numbers are reported both in the text and in tables. This makes difficult to get the underlined points. I suggest to select more relevant data for text.

REPLY: Following the Reviewer's suggestion, the less relevant data have been removed from the text.

The use of M30 is interesting but it is not discussed.

REPLY: M30 data have been reported, together with those of LFT as markers of liver damage. The association of M30 to markers of oxidative stress in the pathogenesis of NASH has been extensively discussed in a previous paper by our group ( Del Ben M et al. Serum cytokeratin-18 is associated to NOX2genenerated oxidative stress in patients with non-alcoholic fatty liver. Int J Hepatol. 2014;2014:784985. doi: 10.1155/2014/784985. Epub 2014 Jan 29.) 
Major points.

The presented data confirms that great correlation do exit between the amount of steatosis and all the components of METs. It is expected that MM could be associated with less extended steatosis. This should be discussed. Apparently patients with abnormal liver tests have been excluded (normal values are not reported in the text), so this population seems not the right choice to address one of the key point of the study: differentiating cardiovascular risk from liver disease risk: it is suggested that MM population might have lover cardiovascular risk (data support this) and possibly higher liver disease risk (not supported by data).,A group of patients with steatosis and liver disease (using as surrogate marker increased ALT) should be added.

REPLY: We confirm that, as reported in the Methods, this is a study performed in consecutive patients referred to our metabolic outpatient clinic who had evidence of fatty liver disease at a liver US performed as part of routine clinical examination. Therefore, patients with abnormal liver tests were not excluded. 


\section{Non-alcoholic fatty liver disease, metabolic syndrome and patatin-like phospholipase domain-containing protein3 (PNPLA3) gene variants}

Short title: NAFLD and PNPLA3 I148M polymorphism

M. Del Ben ${ }^{1}$, L. Polimeni ${ }^{1}$, M. Brancorsini ${ }^{1}$, A. Di Costanzo ${ }^{1}$, L. D’Erasmo ${ }^{1}$, F.

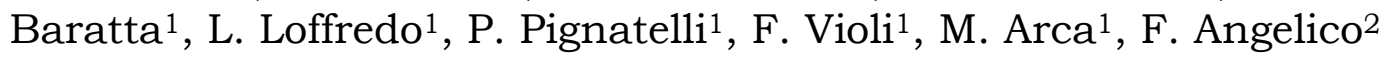

${ }^{1}$ Department of Internal Medicine and Medical Specialties, Sapienza University, Rome, Italy

2 Department of Public Health and Infectious Disease, Sapienza University, Rome, Italy

Abbreviations: Non-alcoholic fatty liver disease (NAFLD); Non-alcoholic steatohepatitis (NASH); patatin-like phospholipase domain-containing protein3 (PNPLA3); Alanine aminotransferase (ALT); Homeostasis model of insulin resistance (HOMA-IR); Metabolic syndrome (MetS); Ultrasonographic scanning (US); Body mass index (BMI); Adiponectin (APN)

\section{Corresponding Author:}

Prof. Francesco Angelico

Department of Public Health and Infectious Disease, Sapienza University, Rome, Italy I Clinica Medica - Policlinico Umberto I, Viale del Policlinico 155, 00161 Rome, Italy Phone/fax +39 9649972249, Email: francesco.angelico@uniroma1.it

Authors' contribution: MDB wrote the manuscript and researched data. LP, FB and LD contributed to data collection, analysis and interpretation; LL and PP reviewed the manuscript; MB and $\mathrm{AD}$ contributed to data collection; MA contributed to study design and reviewed the manuscript; FV reviewed and edited the manuscript. FA designed the study and wrote the manuscript; he is the guarantor of this work and, as such, had full access to all the data in the study and takes responsibility for the integrity of the data and the accuracy of the data analysis. All Authors approved the final manuscript.

Word count main text: 2.588

Word count abstract: 209

No. of tables: 4 
Abstract:

Background \& Aims: Non-alcoholic fatty liver disease was traditionally interpreted as a condition which may progress to liver-related complications. However, the increased mortality is primarily a result of cardiovascular diseases. It has been suggested that fatty liver can be considered as the hepatic consequence of the metabolic syndrome. The aim was to describe the different clinical presentations of non-alcoholic fatty liver disease on the basis of the patatin-like phospholipase domain-containing protein3 (PNPLA3) rs738409 gene variant.

Methods Fatty liver was defined by ultasonographic Hamaguchi's criteria in 211 consecutive subjects with non-alcoholic fatty liver disease. The rs 738409 polymorphism was determined by TaqMan assays. Metabolic syndrome was defined according to ATPIII modified criteria.

Results Prevalence of PNPLA3-148II, PNPLA3-148IM, and PNPLA3-148MM genotypes was $45.0 \%, 40.7 \%$, and $14.3 \%$ respectively. Prevalence of metabolic syndrome progressively increased with the severity of liver steatosis (from $52.5 \%$ to $65.2 \%$, and $82.3 \%$ respectively, $\mathrm{p}<0.01$ ). The PNPLA3-148MM group had significantly lower mean serum triglycerides $(\mathrm{p}<0.001)$, Framingham cardiovascular risk score $(\mathrm{p}<0.01)$ and lower prevalence of metabolic syndrome $(\mathrm{p}<0.05)$ and its components. Age and HOMA-IR were positive independent predictors of metabolic syndrome, while a negative independent association was found between metabolic syndrome and the homozygotes PNPLA3 I148M variant.

Conclusions We suggest a lower prevalence of MetS and reduced cardiovascular risk in NAFLD patients with PNPLA3MM genotype.

Keywords: Non alcoholic fatty liver disease, Metabolic Syndrome, PNPLA3. 


\section{Introduction}

Non-alcoholic fatty liver disease (NAFLD) is the most common and emerging liver disease in Western countries [1,2]. Fatty liver includes a wide spectrum of histologic alterations ranging from simple steatosis, to non-alcoholic steatohepatitis (NASH), which is characterized by inflammation and fibrosis. Moreover, NAFLD has been traditionally interpreted as a condition, which may eventually progress to liver related complications such as cirrhosis, liver cancer and liver mortality [3-5].

However, the pathogenesis of NAFLD is multifactorial and many mechanisms that cause fatty liver infiltration, inflammation, oxidative stress and fibrosis have been proposed.

The association of liver steatosis with a number of common metabolic conditions and cardiovascular risk factors has been extensively reported. Notably, increased mortality of NAFLD patients is primarily a result of cardiovascular diseases and, to a lesser extent, to liver related diseases [6-12]. It has been suggested that fatty liver can be considered as the hepatic consequence of the metabolic syndrome (MetS), a cluster of metabolic disorders including central obesity, hyperglycemia, arterial hypertension, hypertriglyceridemia and low HDL-cholesterol. Thus, a strong bidirectional association between NAFLD and MetS has been proposed. Insulin resistance, the key feature of MetS, is considered to play a central role in the first stages of fatty liver infiltration [13-15]. However, whether insulin resistance and hyperinsulinemia are components of MetS promoting fatty liver or whether NAFLD itself induces chronic hyperinsulinemia by impaired insulin degradation is still under debate. In addition, not all subjects with MetS will develop NAFLD and not all subjects with NAFLD will develop MetS.

Several lines of evidence clearly indicated that also genetic factors may predispose to NAFLD and among the others a variant (I148M) located at the patatin-like phospholipase domain-containing protein 3 (PNPLA3) gene appears to show the strongest effect. Based on the presence of I148M genotype, two different clinical presentations of NAFLD have been recently proposed [16-23]. The first one is associated to the presence of common I148I allele and characterized by a high 
prevalence of obesity and possibly high cardiovascular risk, whereas the other one is associated to the I148M allele presenting an higher susceptibility to more severe liver histology and liver disease progression.

Aim of the study was to evaluate whether PNPLA3 gene variants may predict different clinical phenotypes in a large series of Italian patients with NAFLD.

\section{Materials and methods}

\section{Study patients}

The study was performed in 211 consecutive patients referred to our metabolic outpatient clinic who had evidence of fatty liver disease at a liver ultrasonographic scanning (US) performed as part of routine clinical examination.

To be eligible for the study, patients had to have fulfilled the following inclusion criteria: no history of current or past excessive alcohol drinking as defined by an average daily consumption of alcohol $>20 \mathrm{~g}$; negative tests for the presence of hepatitis B surface antigen and antibody to hepatitis C virus; absence of history and clinical, biochemical and US findings consistent with cirrhosis and other chronic liver diseases. None of the subjects was taking amiodarone and other drugs known to promote fatty liver disease. Subjects underwent routine clinical and biochemical evaluation. Waist circumference, height and weight were recorded and body mass index (BMI) was calculated as weight $(\mathrm{Kg})$ divided by height $\left(\mathrm{m}^{2}\right)$. Blood pressure was recorded following standard procedures. Diabetes was diagnosed according to the WHO criteria [24]. Subjects taking insulin or oral antidiabetic drugs were considered to have diabetes. According to the modified criteria of the ATP III Expert Panel of the US National Cholesterol Education Program [25], metabolic syndrome was diagnosed on the concomitant presence of at least three of the following five clinical features: waist circumference (central obesity) $>102 \mathrm{~cm}$ in men and $>88 \mathrm{~cm}$ in women, fasting blood glucose $\geq$ $100 \mathrm{mg} / \mathrm{dl}$, triglycerides $\geq 150 \mathrm{mg} / \mathrm{dl}$, HDL-cholesterol $<40 \mathrm{mg} / \mathrm{dl}$ in men and $<50 \mathrm{mg} / \mathrm{dl}$ in women, arterial systolic/diastolic blood pressure $\geq 130 / \geq 85 \mathrm{~mm} / \mathrm{Hg}$. A metabolic score was 
calculated for each patient based on the number of the discrete components of MS identified. Cardiovascular risk was expressed as Framingham score determined by using the National Cholesterol Education Program (NCEP) calculator, available on-line at http://hp2010.nhlbihin.net/atpiii/calculator.asp

Written informed consent was obtained from all patients before the study. The study was approved by the hospital Ethics Committee and conforms to the ethical guidelines of the 1975 Declaration of Helsinki.

\section{Laboratory measurements}

Serum total cholesterol, HDL-cholesterol and triglycerides were measured by an Olympus AN 560 apparatus using an enzymatic colorimetric method. LDL-cholesterol levels were calculated according to the Friedwald formula. Plasma insulin levels were assayed by commercially available radioimmunoassay. The homeostasis model of insulin resistance (HOMA-IR), based on serum fasting glucose and insulin levels, was used as a measure of IR [26].

Adiponectin (APN) serum levels were measured with a commercial immunoassay (Tema Ricerca, Italy). Intra-assay and inter-assay coefficients of variation were 6 and 8\%, respectively. Serum levels of Cytokeratin 18-M30 were measured as marker of liver damage with a commercial immunoassay (Tema Ricerca, Italy) and expressed as mlU/ml. Intra-assay and inter-assay coefficients were $6 \%$ and $7 \%$ respectively.

\section{Ultrasonographic examination}

Liver US scanning was performed to assess the degree of steatosis. All US were performed by the same operator who was blinded to laboratory values using a GE Vivid S6 apparatus equipped with a convex 3,5 MHz probe. Liver steatosis was defined according to Hamaguchi criteria based on the presence of abnormally intense, high level echoes arising from the hepatic parenchyma, liverkidney difference in echo amplitude, echo penetration into deep portion of the liver and clarity of 
liver blood vessel structure [27,28]. Steatosis was assessed semi-quantitatively on a scale of 0-6: 0, absent; 1,2 mild; 3,4 moderate; 5,6 severe.

\section{Genetic analysis}

DNA was extracted from peripheral blood and purified by the The Wizard® Genomic DNA Purification Kit following the manufacturing protocol. Fluorogenic 5'-nucleotidase assays were developed to genotype the PNPLA3 rs $738409 \mathrm{C}$ to $\mathrm{G}$ nonsynonymous sequence variant, encoding I148M, in all subjects. The assay was performed using the TaqMan C7241_10 assay (Applied Biosystems, Foster City, CA) on ABI PRISM 7900 HT Sequence Detection Systems. (Applied Biosystems). The plate was run using standard condition at $95^{\circ} \mathrm{C}$ for $10 \mathrm{~min}, 95^{\circ} \mathrm{C}$ for $15 \mathrm{~s}$ then $60{ }^{\circ} \mathrm{C}$ for $1 \mathrm{~min}$ for 40 cycles. Allele frequencies were in Hardy-Weinberg equilibrium. The TaqMan assay was validated by direct sequencing of the SNP(rs738409) in representative samples of DNA on ABI PRISM 3130 XL Genetic Analyzer, and both methods gave identical results.

\section{Statistical analysis}

Statistical analysis was performed using the SPSS statistical software version $\underline{18.0}$ for Windows (SPSS, Inc., Chicago. Illinois). Student's t-test for unpaired data was used for the comparison of mean values. Distribution of continuous variables was tested for normality using the KolmogorovSmirnov test. Data are expressed as the mean \pm SD for normally distributed variables and as median and interquartile range for non-normally distributed data. Group comparisons were performed by use of analysis of variance (ANOVA) and unpaired Student's t-test when appropriate. Non normally distributed variables were tested by Mann-Whitney test and Kruskall-Wallis test. Proportions and categorical variables were tested by the $\chi^{2}$-test and by the 2-tailed Fisher's exact method when appropriate. All tests were two-tailed; a $P$ value of less than 0.05 was considered to indicate statistically significance. Multivariate analyses were performed using the stepwise logistic regression analysis testing for the dichotomous response variable presence or absence of MetS after 
controlling for possible clinical and biochemical confounders. The predictor variables entered in the different models were age, gender, PNPLA3-148MM, Hamaguchi score, HOMA-IR, serum lipid levels, serum ALT, adiponectin and cytokeratin-18 values, positive family history for diabetes and cardiovascular disease.

\section{Results}

Mean age was $54.5+11.9$ years. Among the 211 subjects with fatty liver, US examination revealed mild steatosis in 40, moderate steatosis in 90 and severe steatosis in 81 . Clinical and laboratory characteristics of subjects (mean age $54.5 \pm 11.9$ yrs) with different severity of fatty liver according to Hamaguchi's classification of steatosis are reported in Table 1. A progressive, statistically significant increase in the mean values of the indexes of central obesity (BMI, waist and hip circumferences), glucose metabolism (fasting blood glucose, insulin, HOMA-IR and glycosylated haemoglobin) serum alanine aminotransferase (ALT) and cytokeratine-18 was observed from the group with mild steatosis to the groups with moderate and severe steatosis; conversely, an opposite trend was observed for serum HDL cholesterol and adiponectin levels. No statistically significant between-group differences were observed for total and LDL cholesterol, triglycerides, aspartate aminotransferase (AST), $\gamma$-glutamyltransferase $(\gamma \mathrm{GT})$, smoking, and statin use.

The prevalence of MetS and its five clinical features defined according to ATPIII criteria is reported in Table 2. Prevalence of MetS progressively increased from subjects with mild steatosis to those with moderate and severe steatosis (from $52.5 \%$ to $65.2 \%$ and $82,3 \%$ respectively, $\mathrm{p}<0.01$ ). Compared to subjects with mild steatosis, the relative risk in those with severe steatosis was 1.3 (C.I. 1.0-1.6, $\mathrm{p}<0.02$ ) for increased waist circumference, 1.6 (C.I. 1.2-2.2, $\mathrm{p}<0.01$ ) for high blood pressure, 2.6 (C.I. 1.5-4.6, p<0.01) for high fasting glucose, 1.4 (C.I. 0.9-2.1 p=013) for high triglycerides and 1.9 (C.I. 1.0-3.4, p<0.05) for low HDL cholesterol. Clinical and biochemical variables of subjects with fatty liver according to the different PNPLA3 genotypes are reported in Table 3. The overall prevalence of PNPLA3-148II, PNPLA3-148IM and 
PNPLA3-148MM genotypes in our cohort was $45.0 \%(n=95), 40.7 \%(n=86)$ and $14.3 \%(n=30)$ respectively. A progressive, statistically significant decrease of mean serum triglycerides, Framingham cardiovascular risk score (from 9 (3/16.7) to 6 (2/13) and 4 (2/8.2) \% respectively, $\mathrm{p}<0.01$ ), MetS score and of the prevalence of MetS (from $71.6 \%$ to $72.1 \%$ and $50 \%$ respectively, $\mathrm{p}<0.05)$ was observed from the group with II genotype to those with genotypes IM and MM. The different groups were comparable with respect to age, BMI, waist circumference, US Hamaguchi score, serum total and LDL-cholesterol, GGT and cytokeratin-18.

Since at univariate analysis a lower prevalence of MetS was present in MM carriers, several models were generated by stepwise logistic analyses to assess the independent contribution of MM genotype for the prediction of MetS. In a stepwise logistic analysis including 10 clinical and biochemical covariates [Table 4], age, HOMA-IR, and the Hamaguchi score for steatosis were positive independent predictors of MetS, while a negative independent association was found between MetS and the homozygotes PNPLA3-I148M variant (overall predictive value of the model: $\left.\mathrm{R}^{2}=0.30\right)$.

\section{Discussion}

Our results show for the first time that PNPLA3-I148M gene variant is associated with a lower prevalence of MetS and reduced cardiometabolic risk in a large series of individuals with NAFLD. In fact, univariate and multivariate analyses suggest that individuals carrying the PNPLA3 MM genotype have a lower probability of having MetS.

Recently, the widely accepted assumption that NAFLD should be always considered the hepatic manifestation of MetS has been disputed and the study of PNPLA3-I148M polymorphism has been proposed as a useful tool to discriminate fatty liver with and without metabolic consequences and for estimating the risk for future cardiovascular or liver disease [18-19]. In fact, although the mechanisms underlining the association between PNPLA3 polymorphisms and NAFLD are still 
unclear, the I148M polymorphism seems to be associated to increased hepatic lipid accumulation and more severe histological liver disease progression [17,20-22].

Few studies have been performed to assess the different clinical phenotypes associated to PNPLA3 polymorphism and none referring to MetS as a clinical condition diagnosed according to standardized criteria; all refer only to some aspects of MetS; most contain small number of patients and report conflicting results.

In a recent study subjects homozygotes for the variant allele (PNPLA3-148MM) had significantly increased liver fat content with no insulin and triglyceride elevation, nor adipose tissue inflammation [16]. In a multi-ethnic study performed in Malaysia, where PNPLA3-I148M allele variation was associated with NASH susceptibility, NASH severity and the presence of fibrosis at liver histology, but not with simple steatosis and hypertriglyceridemia [17]. A further study performed in 335 individuals in Germany showed that PNPLA3-148MM genotype is strongly and independently associated with fatty liver but not with insulin resistance or estimates of liver injury [18]. In another study performed in Hispanic children and adolescents, liver fat in 148MM carriers was 2.7 times higher at nuclear resonance imaging than in wild type carriers and children with 148MM genotype had significantly lower HDL-cholesterol levels [19]. Moreover, a large study showed that variation at PNPLA3 confers a markedly increased risk of increasingly severe histological features of NAFLD, without a strong effect on some components of MetS [20]. Finally, a meta-analysis performed on 16 studies across different populations showed a strong influence of PNPLA3-I148M variation not only on liver fat accumulation, but also on the susceptibility of a more aggressive disease, with MM carriers having a greater risk of higher necro-inflammation and of developing fibrosis when compared with PNPLA3-I148I homozygotes [21].

By contrast, in a large study performed in a normoglycaemic population of Taiwan, PNPLA3148MM genotype was strongly associated with NAFLD and subjects had increased waist circumference and higher fasting insulin concentrations and higher HOMA-IR and ALT concentrations [29]. The reasons for these conflicting results are unclear and possibly depending on 
the ethnic differences on the relative role of insulin resistance and MetS in the pathogenesis of NAFLD [30].

In our study, NAFLD patients with PNPLA3-148MM genotype had a lower prevalence of MetS, which may suggest a lower global cardiovascular risk in PNPLA3-148MM carriers. Notably, low levels of serum triglycerides mainly accounted for the lower prevalence of MetS in PNPLA3MM subjects. These findings were also confirmed by the lower average calculated Framingham cardiovascular risk score in MM homozygotes as compared to wild type carriers. Our results are consistent with the hypothesis that NAFLD might have two different clinical presentations, one associated to the common PNPLA3-I148I allele, presenting the clinical phenotype of MetS, insulin resistance and high cardiovascular risk, and the other, less frequent, associated to the PNPLA3-I148M allele, with a higher susceptibility to more severe liver histology and liver disease progression. Finally, our findings may also suggest that in the future genetic PNPLA3 screening could become an attractive novel target to identify individuals at high risk for cardio-metabolic diseases or for progressive liver disease.

The decreased triglyceridaemia found in our PNPLA3-148MM carriers is consistent with the results of a study showing a lower VLDL secretion in the liver of PNPLA3-148MM carriers, as compared to the PNPLA3-148II homozygotes, with PNPLA3-148MM genotype alone explaining 46\% of the variation in of VLDL triglyceride secretion. The Authors suggest that this might be the result of a reduced triglyceride hydrolysis in the adipose tissue leading to decreased VLDL synthesis and secretion and subsequent lipid deposition in the liver [31].

Our study may have some limitations. First, the study has been performed in patients recruited in a hospital-based setting. Second, fatty liver was assessed by US; the gold standard for the diagnosis of NAFLD is liver biopsy, but this is an invasive procedure with potentially serious complications and is therefore not acceptable without clinical indication. We acknowledge that grades of steatosis could have been better determined by magnetic resonance spectroscopy. However, Hamaguchi score showed $100 \%$ specificity and $91.7 \%$ sensitivity when compared with liver biopsy in NAFLD 
patients [26]. A further limitation is the higher prevalence of females in PNPLA3MM group which may have determined the lower cardiometabolic risk, considering the weight that gender has in the Framingham score. Finally, the rather low number of patients in the PNPLA3MM group may also represent a limitation to draw definitive conclusions based on genetic polymorphism.

\section{Conclusions}

We suggest that NAFLD may not always represent the hepatic manifestation of MetS. In fact, in our study, patients with NAFLD carrying PNPLA3-I148M gene had a lower prevalence of MetS and lower cardiometabolic risk. Further studies are needed to confirm the possible role of PNPLA3 polymorphisms to differentiate clinical phenotypes of NAFLD and to discriminate NAFLD with possible metabolic and cardiovascular consequences from fatty liver at risk for liver related complications.

Conflict of interests: All authors declare that they have no conflicts of interest.

\section{Learning points:}

- NAFLD does not always represent the hepatic manifestation of MetS

- PNPLA3 I148M gene variant is associated with a lower prevalence of metabolic syndrome and lower cardiometabolic risk. 


\section{References}

1. Ruhl CE, Everhart JE. Epidemiology of nonalcoholic fatty liver. Clin Liver Dis 2004; 8:501-19.

2. Bedogni G, Miglioli L, Masutti F, Tiribelli C, Marchesini G, Bellentani S. Prevalence of risk factors for non-alcoholic fatty liver disease: the Dionysos nutrition and liver study. Hepatology 2005; 42: 44-52

3. Neuschwander-Tetri BA, Caldwell SH. Nonalcoholic steatohepatitis: summary of an AASLD Single Topic Conference. Hepatology 2003; 37: 1202-19

4. Angulo P. Nonalcoholic fatty liver disease. N Engl J Med 2002; 346: 1221-31.

5. Day CP. Pathogenesis of steatohepatitis. Best Pract Res Clin Gastroenterol 2002; 16: 66378

6. Despres JP, Lemieux I, Bergeron J et al. Abdominal obesity and the metabolic syndrome: contribution to global cardiometabolic risk. Arterioscler Thromb Vasc Biol 2008; 28: 103949

7. Mottillo $\mathrm{S}$, Filion $\mathrm{KB}$, Genest $\mathrm{J}$ et al. The metabolic syndrome and cardiovascular risk: a systematic review and meta-analysis. J Am Coll Cardiol 2010; 56: 1113-32

8. Targher G, Day CP, Bonora E. Risk of cardiovascular disease in patients with nonalcoholic fatty liver disease. N Engl J Med 2010; 363: 1341-50

9. Del Ben M, Baratta F, Polimeni L, Angelico F. Non-alcoholic fatty liver disease and cardiovascular disease: epidemiological, clinical and pathophysiological evidences. Intern Emerg Med 2012; 7 (Suppl 3): S291-S296

10. Targher G, Marra F, Marchesini G. Increased risk of cardiovascular disease in nonalcoholic fatty liver disease: causal effect or epiphenomenon? Diabetologia 2008; 51:1947-53.

11. Lin YC, Lo HM, Chen JD. Sonographic fatty liver, overweight and ischemic heart disease. World J Gastroenterol 2005; 11:4838-42.

12. Mirbagheri SA, Rashidi A, Abdi S, Saedi D, Abouzari M. Liver: an alarm for the heart? Liver Int 2007; 27: 891-4.

13. Angelico F, Del Ben M, Conti R et al. Non alcoholic fatty liver disease: an hepatic consequence of common metabolic diseases. J Gastroenterol Hepatol 2003;18: 588-94.

14. Kotronen A, Yki-Järvinen H. Fatty liver: a novel component of the metabolic syndrome. Arterioscler Thromb Vasc Biol 2008; 28: 27-38.

15. Angelico F, Del Ben M, Conti R et al. Insulin resistance, the metabolic syndrome, and nonalcoholic fatty liver disease. J Clin Endocrinol Metab 2005; 90: 1578-82 
16. Lallukka S, Sevastianova K, Perttilä J et al. Adipose tissue is inflamed in NAFLD due to obesity but not in NAFLD due to genetic variation in PNPLA3. Diabetologia 2013; 56: 88692

17. Zain SM, Mohamed R, Mahadeva S et al. A multi-ethnic study of a PNPLA3 gene variant and its association with disease severity in non-alcoholic fatty liver disease. Hum Genet 2012; 131: 1145-52

18. Kantartzis K, Peter A, Machicao F et al. Dissociation between fatty liver and insulin resistance in humans carrying a variant of the patatin-like phospholipase 3 gene. Diabetes 2009; 58: 2616-2

19. Goran MI, Walker R, Le KA et al. Effects of PNPLA3 on liver fat and metabolic profile in Hispanic children and adolescents. Diabetes 2010; 59: 3127-3

20. Speliotes EK, Butler JL, Palmer CD et al. PNPLA3 variants specifically confer increased risk for histologic nonalcoholic fatty liver disease but not metabolic disease. Hepatology 2010; 52: 904-912

21. Sookoian S, Pirola CJ. Meta-analysis of the influence of I148M variant of patatin-like phospholipase domain containing 3 gene (PNPLA3) on the susceptibility and histological severity of nonalcoholic fatty liver disease. Hepatology 2011; 53: 1883-94

22. Dubuquoya C, Burnola AF, Moldesa M. PNPLA3, a genetic marker of progressive liver disease, still hiding its metabolic function? Clin Res Hepatol Gastroenterol 2012; 37: 30-5

23. Lallukka S, Sevastianova K, Perttila J, et al. Adipose tissue is inflamed in NAFLD due to obesity but not in NAFLD due to genetic variation in PNPLA3 Diabetologia 2013; 56: 88692.

24. American Diabetes Association. Diagnosis and classification of diabetes mellitus. Diabetes Care 2011; 34(Suppl. 1): S62-S69

25. Grundy SM, Cleeman JI, Daniels SR et al. Diagnosis and management of the metabolic syndrome: an American Heart Association/National Heart, Lung, and Blood Institute Scientific Statement. Circulation 2005; 112: 2735-52

26. Matthews DR, Hosker JP, Rudenski AS, Naylor BA, Treacher DF, Turner RC. Homeostasis model assessment: insulin resistance and beta-cell function from fasting plasma glucose and insulin concentrations in man. Diabetologia, 1985; 28: 412-19

27. Hamaguchi M, Kojima T, Itoh Y et al. The severity of ultrasonographic findings in nonalcoholic fatty liver disease reflects the metabolic syndrome and visceral fat accumulation. Am J Gastroenterol 2007; 102: 2708-15.

28. Saverymuttu SH, Joseph AEA, Maxwell JD. Ultrasound scanning in the detection of hepatic fibrosis and steatosis. Br Med J 1986; 292: 13-15

29. Wang CW, Lin HY, Shin SJ et al. The PNPLA3 I148M polymorphism is associated with insulin resistance and nonalcoholic fatty liver disease in a normoglycaemic population Liver Int 2011; 31: 1326-31 
30. Chang CY. Understanding the relationship between PNPLA3, NAFLD and insulin resistance: do ethnic differences bring more questions or more answers? Liver Int 2011; 31: 1246-1249

31. Pirazzi C, Adiels M, Burza MA et al. Patatin-like phospholipase domain-containing 3 (PNPLA3) I148M (rs738409) affects hepatic VLDL secretion in humans and in vitro. J Hepatol 2012; 57: 1276-82 
Table 1. Clinical and biochemical characteristics of subjects according to severity of nonalcoholic fatty liver disease (NAFLD) at ultrasonography

\begin{tabular}{|c|c|c|c|c|}
\hline & \multicolumn{3}{|c|}{ NAFLD } & \multirow[b]{2}{*}{$\mathbf{p}$} \\
\hline & $\begin{array}{c}\text { Mild } \\
(n=40)\end{array}$ & $\begin{array}{c}\text { Moderate } \\
\quad(n=90)\end{array}$ & $\begin{array}{c}\text { Severe } \\
(n=81)\end{array}$ & \\
\hline $\begin{array}{l}\text { Gender }(\mathbf{F} / \mathbf{M}) \% \\
\text { Age }(\mathrm{yrs}) \\
\text { BMI }(\mathrm{kg} / \mathrm{m} 2) \\
\text { Waist circumference }(\mathrm{cm})\end{array}$ & $\begin{array}{c}45.0 / 55.0 \\
49.9(14.6) \\
29.6(5.1) \\
103,5(97,3 / 109,5)\end{array}$ & $\begin{array}{c}36.7 / 63.3 \\
56.0(11.5) \\
30.3(5.3) \\
105(98 / 113)\end{array}$ & $\begin{array}{c}29.6 / 70.4 \\
54.6(10.4) \\
33.5(5.3) \\
113(106 / 125)\end{array}$ & $\begin{array}{l}\mathrm{ns} \\
\mathrm{ns} \\
<.001 \\
<.001\end{array}$ \\
\hline $\begin{array}{l}\text { Fasting plasma glucose }(\mathrm{mg} / \mathrm{dl}) \\
\text { Insulin }(\mathrm{mU} / \mathrm{l}) \\
\text { HbA1c }(\%) \\
\text { HOMA IR } \\
\text { Total Cholesterol }(\mathrm{mg} / \mathrm{dl}) \\
\text { Triglycerides }(\mathrm{mg} / \mathrm{dl}) \\
\text { HDL cholesterol }(\mathrm{mg} / \mathrm{dl}) \\
\text { LDL cholesterol }(\mathrm{mg} / \mathrm{dl}) \\
\text { AST }(\mathrm{UI} / \mathrm{l}) \\
\text { ALT }(\mathrm{UI} / \mathrm{l}) \\
\gamma \text { GT }(\mathrm{UI} / \mathrm{l}) \\
\text { Cytokeratin } 18(\mathrm{mIU} / \mathrm{ml}) \\
\text { Adiponectin }(\mathrm{ng} / \mathrm{ml}) \\
\text { Framingham cardiovascular } \\
\text { risk score }(\%)^{*}\end{array}$ & $\begin{array}{c}92(86 / 100,5) \\
11,5(7,7 / 15,5) \\
5,4(5,1 / 5,7) \\
2.5(1.6 / 3.7) \\
209(41) \\
132,5(188,3 / 175 / 8) \\
53(43 / 60) \\
125(34) \\
19(16 / 26,8) \\
21(17,3 / 34) \\
23(16,5 / 42,3) \\
169,5(129,3 / 183,8) \\
10,3(5,5 / 12) \\
5(1 / 14,5)\end{array}$ & $\begin{array}{c}97(92,8 / 117,5) \\
13(9,3 / 17,9) \\
5,6(5,3 / 6,1) \\
3.1(2.2 / 4.7) \\
201(40) \\
138(99,8 / 179) \\
45,5(39,8 / 60,3) \\
121(35) \\
21(18 / 25) \\
26(20,8 / 40) \\
21(16 / 36) \\
176(140 / 190) \\
8,8(6 / 13,1) \\
6(3 / 13)\end{array}$ & $\begin{array}{c}107(95 / 128,3) \\
16,6(12,5 / 24,5) \\
6(5,6 / 6,5) \\
5.2(3.1 / 7.3) \\
198(40) \\
152,5(119,5 / 197,8) \\
43(38 / 51) \\
118(33) \\
22(18 / 32) \\
34(24 / 45) \\
32(23,8 / 55) \\
180(169,5 / 192,5) \\
6,0(4,9 / 9,1) \\
3(9 / 14)\end{array}$ & $\begin{array}{l}<.001 \\
<.001 \\
<.001 \\
<.001 \\
\mathrm{~ns} \\
\mathrm{~ns} \\
<.05 \\
\mathrm{~ns} \\
\mathrm{~ns} \\
<.05 \\
\mathrm{~ns} \\
<.01 \\
<.001 \\
\mathrm{~ns}\end{array}$ \\
\hline *see Methods & & & & \\
\hline
\end{tabular}


Table 2. Prevalence of MetS and its clinical features in subjects with different severity of steatosis at liver ultrasonography.

\begin{tabular}{l|c|c|c|c}
\hline & \multicolumn{3}{|c|}{ NAFLD } & \\
\cline { 2 - 5 } & $\begin{array}{c}\text { Mild } \\
(\mathrm{n}=40)\end{array}$ & $\begin{array}{c}\text { Moderate } \\
(\mathrm{n}=90)\end{array}$ & $\begin{array}{c}\text { Severe } \\
(\mathrm{n}=81)\end{array}$ & $\mathbf{p}$ \\
\hline Metabolic Syndrome (\%)* & 52.5 & 65.2 & 82.3 & $<.01$ \\
High blood pressure (\%)* & 55 & 78.9 & 88.6 & $<.001$ \\
High waist circumference (\%)* & 70 & 75 & 91.1 & $<.01$ \\
High fasting blood glucose (\%)* & 25 & 44.4 & 65 & $<.001$ \\
High triglycerides (\%)* & 40 & 44.4 & 55 & $\mathrm{~ns}$ \\
Low HDL-cholesterol (\%)* & 25 & 35.6 & 46.3 & $\mathrm{~ns}$ \\
MetS Score** & $3(2 / 4)$ & $3(2 / 4)$ & $4(3 / 5)$ & $<.01$ \\
\hline
\end{tabular}

*Defined according to ATPIII criteria; **see Methods 
Table 3. Clinical and biochemical characteristics of subjects with different PNPLA3 genotypes

\begin{tabular}{|c|c|c|c|c|}
\hline \multicolumn{5}{|c|}{ PNPLA3 genotypes } \\
\hline & $\begin{array}{l}\text { 148II } \\
(95)\end{array}$ & $\begin{array}{l}\text { 148IM } \\
(86)\end{array}$ & $\begin{array}{l}\text { 148MM } \\
\text { (30) }\end{array}$ & $\mathbf{p}$ \\
\hline Gender (F/M) \% & $27.4 / 72.6$ & $39.5 / 60.5$ & $50 / 50$ & $<.05^{*}$ \\
\hline Metabolic Syndrome (\%) & 71.6 & 72.1 & 50 & $<.05 *$ \\
\hline Age (yrs) & $54 \pm 11$ & $54 \pm 12.8$ & $54.3 \pm 12.1$ & ns \\
\hline Weight $(\mathrm{kg})$ & $92.2 \pm 18.1$ & $89.0 \pm 16.5$ & $89.8 \pm 16.2$ & ns \\
\hline BMI $(\mathrm{kg} / \mathrm{m} 2)$ & $32.1 \pm 5.6$ & $30.4 \pm 5.1$ & $31.7 \pm 6.1$ & ns \\
\hline Waist circumference $(\mathrm{cm})$ & $110(103.5 / 118)$ & $105(101 / 113.5)$ & $106(96.7 / 118.5)$ & ns \\
\hline Fasting plasma glucose $(\mathrm{mg} / \mathrm{dl})$ & $101(92 / 110)$ & $97(92 / 121)$ & $101(87.5 / 128.2)$ & ns \\
\hline Insulin $(\mu \mathrm{U} / \mathrm{ml})$ & $14.1(10.7 / 20.2)$ & $13.7(10.1 / 19.4)$ & $11.6(8.7 / 16.8)$ & ns \\
\hline HOMA IR & $3.6(2.3 / 5.8)$ & $3.5(2.5 / 5.7)$ & $2.8(2.0 / 5.6)$ & ns \\
\hline Total Cholesterol (mg/dl) & $205 \pm 41.9$ & $198 \pm 40.3$ & $196.9 \pm 34.3$ & $\mathrm{~ns}$ \\
\hline Triglycerides (mg/dl) & $157(112 / 193)$ & 141(107.7/185) & 111.5(90.7/148.7) & $<.01 *$ \\
\hline HDL cholesterol (mg/dl) & $45(39 / 53)$ & $44(38 / 56)$ & $50.5(43 / 61)$ & $\mathrm{ns}$ \\
\hline AST (UI/l) & $20(17 / 25)$ & $21(18 / 31)$ & $23(17.7 / 27.7)$ & $\mathrm{ns}$ \\
\hline ALT (UI/l) & $26(19 / 35)$ & $30.5(22 / 45)$ & $30(22.7 / 40.5)$ & ns \\
\hline$\gamma \mathbf{G T}(\mathrm{UI} / \mathrm{l})$ & $28(19 / 42)$ & $28(17 / 46)$ & $22(17.5 / 39.5)$ & $\mathrm{ns}$ \\
\hline Cytokeratine $18(\mathrm{mIU} / \mathrm{ml})$ & $180(143 / 192)$ & $180(151.2 / 190)$ & $170.5(130 / 182.2)$ & ns \\
\hline Adiponectin (ng/ml) & $7.5(5 / 11)$ & $7.5(5.5 / 12)$ & $9.3(4.9 / 13.1)$ & ns \\
\hline $\begin{array}{l}\text { Framingham cardiovascular } \\
\text { risk score }(\%)\end{array}$ & $9(3 / 16.7)$ & $6(2 / 13)$ & $4(2 / 8.2)$ & $<.01 *$ \\
\hline Hamaguchi score & $4(3 / 5)$ & $4(3 / 5)$ & $4(3 / 5)$ & $\mathrm{ns}$ \\
\hline MetS score ${ }^{* *}$ & $4(2 / 5)$ & $3(2 / 4)$ & $2(1,75 / 4)$ & $<.01 *$ \\
\hline
\end{tabular}

*148MM vs 148II; **see Methods 
Table 4. Multiple logistic analysis of independent predictors of metabolic syndrome in 211 subjects with non-alcoholic fatty liver disease.

\begin{tabular}{lcccccc} 
& B & S.E. & P. & \multicolumn{2}{c}{ O.R. } & \multicolumn{2}{c}{$\mathbf{9 5 , 0 \%}$ C.I. for O.R. } \\
\cline { 2 - 7 } Age & & & & & Lower & Upper \\
PNPLA3.148MM & .062 & .015 & .000 & 1.064 & 1.032 & 1.096 \\
HOMA-IR & -1.195 & .460 & .009 & .303 & .123 & .746 \\
Constant & .267 & .083 & .001 & 1.306 & 1.110 & 1.538 \\
& -3.234 & .858 & .000 & .039 & &
\end{tabular}

Variables entered on step 1: age, gender, PNPLA3.148MM, Hamaguchi score, HOMA-IR, serum ALT serum adiponectin, positive family history for diabetes and cardiovascular disease. 


\section{Non-alcoholic fatty liver disease, metabolic syndrome and patatin-like phospholipase domain-containing protein3 (PNPLA3) gene variants}

Short title: NAFLD and PNPLA3 I148M polymorphism

M. Del Ben ${ }^{1}$, L. Polimeni ${ }^{1}$, M. Brancorsini ${ }^{1}$, A. Di Costanzo ${ }^{1}$, L. D’Erasmo $^{1}$, F.

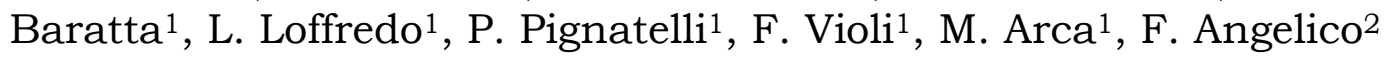

${ }^{1}$ Department of Internal Medicine and Medical Specialties, Sapienza University, Rome, Italy

2 Department of Public Health and Infectious Disease, Sapienza University, Rome, Italy

Abbreviations: Non-alcoholic fatty liver disease (NAFLD); Non-alcoholic steatohepatitis (NASH); patatin-like phospholipase domain-containing protein3 (PNPLA3); Alanine aminotransferase (ALT); Homeostasis model of insulin resistance (HOMA-IR); Metabolic syndrome (MetS); Ultrasonographic scanning (US); Body mass index (BMI); Adiponectin (APN)

\section{Corresponding Author:}

Prof. Francesco Angelico

Department of Public Health and Infectious Disease, Sapienza University, Rome, Italy I Clinica Medica - Policlinico Umberto I, Viale del Policlinico 155, 00161 Rome, Italy Phone/fax +39 9649972249, Email: francesco.angelico@uniroma1.it

Authors' contribution: MDB wrote the manuscript and researched data. LP, FB and LD contributed to data collection, analysis and interpretation; LL and PP reviewed the manuscript; MB and $\mathrm{AD}$ contributed to data collection; MA contributed to study design and reviewed the manuscript; FV reviewed and edited the manuscript. FA designed the study and wrote the manuscript; he is the guarantor of this work and, as such, had full access to all the data in the study and takes responsibility for the integrity of the data and the accuracy of the data analysis. All Authors approved the final manuscript.

Word count main text: 2.588

Word count abstract: 209

No. of tables: 4 
Abstract:

Background \& Aims: Non-alcoholic fatty liver disease was traditionally interpreted as a condition which may progress to liver-related complications. However, the increased mortality is primarily a result of cardiovascular diseases. It has been suggested that fatty liver can be considered as the hepatic consequence of the metabolic syndrome. The aim was to describe the different clinical presentations of non-alcoholic fatty liver disease on the basis of the patatin-like phospholipase domain-containing protein3 (PNPLA3) rs738409 gene variant.

Methods Fatty liver was defined by ultasonographic Hamaguchi's criteria in 211 consecutive subjects with non-alcoholic fatty liver disease. The rs738409 polymorphism was determined by TaqMan assays. Metabolic syndrome was defined according to ATPIII modified criteria.

Results Prevalence of PNPLA3-148II, PNPLA3-148IM, and PNPLA3-148MM genotypes was $45.0 \%, 40.7 \%$, and $14.3 \%$ respectively. Prevalence of metabolic syndrome progressively increased with the severity of liver steatosis (from $52.5 \%$ to $65.2 \%$, and $82.3 \%$ respectively, $\mathrm{p}<0.01$ ). The PNPLA3-148MM group had significantly lower mean serum triglycerides $(\mathrm{p}<0.001)$, Framingham cardiovascular risk score $(\mathrm{p}<0.01)$ and lower prevalence of metabolic syndrome $(\mathrm{p}<0.05)$ and its components. Age and HOMA-IR were positive independent predictors of metabolic syndrome, while a negative independent association was found between metabolic syndrome and the homozygotes PNPLA3 I148M variant.

Conclusions We suggest a lower prevalence of MetS and reduced cardiovascular risk in NAFLD patients with PNPLA3MM genotype.

Keywords: Non alcoholic fatty liver disease, Metabolic Syndrome, PNPLA3. 


\section{Introduction}

Non-alcoholic fatty liver disease (NAFLD) is the most common and emerging liver disease in Western countries [1,2]. Fatty liver includes a wide spectrum of histologic alterations ranging from simple steatosis, to non-alcoholic steatohepatitis (NASH), which is characterized by inflammation and fibrosis. Moreover, NAFLD has been traditionally interpreted as a condition, which may eventually progress to liver related complications such as cirrhosis, liver cancer and liver mortality [3-5].

However, the pathogenesis of NAFLD is multifactorial and many mechanisms that cause fatty liver infiltration, inflammation, oxidative stress and fibrosis have been proposed.

The association of liver steatosis with a number of common metabolic conditions and cardiovascular risk factors has been extensively reported. Notably, increased mortality of NAFLD patients is primarily a result of cardiovascular diseases and, to a lesser extent, to liver related diseases [6-12]. It has been suggested that fatty liver can be considered as the hepatic consequence of the metabolic syndrome (MetS), a cluster of metabolic disorders including central obesity, hyperglycemia, arterial hypertension, hypertriglyceridemia and low HDL-cholesterol. Thus, a strong bidirectional association between NAFLD and MetS has been proposed. Insulin resistance, the key feature of MetS, is considered to play a central role in the first stages of fatty liver infiltration [13-15]. However, whether insulin resistance and hyperinsulinemia are components of MetS promoting fatty liver or whether NAFLD itself induces chronic hyperinsulinemia by impaired insulin degradation is still under debate. In addition, not all subjects with MetS will develop NAFLD and not all subjects with NAFLD will develop MetS.

Several lines of evidence clearly indicated that also genetic factors may predispose to NAFLD and among the others a variant (I148M) located at the patatin-like phospholipase domain-containing protein 3 (PNPLA3) gene appears to show the strongest effect. Based on the presence of I148M genotype, two different clinical presentations of NAFLD have been recently proposed [16-23]. The first one is associated to the presence of common I148I allele and characterized by a high 
prevalence of obesity and possibly high cardiovascular risk, whereas the other one is associated to the I148M allele presenting an higher susceptibility to more severe liver histology and liver disease progression.

Aim of the study was to evaluate whether PNPLA3 gene variants may predict different clinical phenotypes in a large series of Italian patients with NAFLD.

\section{Materials and methods}

\section{Study patients}

The study was performed in 211 consecutive patients referred to our metabolic outpatient clinic who had evidence of fatty liver disease at a liver ultrasonographic scanning (US) performed as part of routine clinical examination.

To be eligible for the study, patients had to have fulfilled the following inclusion criteria: no history of current or past excessive alcohol drinking as defined by an average daily consumption of alcohol $>20 \mathrm{~g}$; negative tests for the presence of hepatitis B surface antigen and antibody to hepatitis C virus; absence of history and clinical, biochemical and US findings consistent with cirrhosis and other chronic liver diseases. None of the subjects was taking amiodarone and other drugs known to promote fatty liver disease. Subjects underwent routine clinical and biochemical evaluation. Waist circumference, height and weight were recorded and body mass index (BMI) was calculated as weight $(\mathrm{Kg})$ divided by height $\left(\mathrm{m}^{2}\right)$. Blood pressure was recorded following standard procedures. Diabetes was diagnosed according to the WHO criteria [24]. Subjects taking insulin or oral antidiabetic drugs were considered to have diabetes. According to the modified criteria of the ATP III Expert Panel of the US National Cholesterol Education Program [25], metabolic syndrome was diagnosed on the concomitant presence of at least three of the following five clinical features: waist circumference (central obesity) $>102 \mathrm{~cm}$ in men and $>88 \mathrm{~cm}$ in women, fasting blood glucose $\geq$ $100 \mathrm{mg} / \mathrm{dl}$, triglycerides $\geq 150 \mathrm{mg} / \mathrm{dl}$, HDL-cholesterol $<40 \mathrm{mg} / \mathrm{dl}$ in men and $<50 \mathrm{mg} / \mathrm{dl}$ in women, arterial systolic/diastolic blood pressure $\geq 130 / \geq 85 \mathrm{~mm} / \mathrm{Hg}$. A metabolic score was 
calculated for each patient based on the number of the discrete components of MS identified. Cardiovascular risk was expressed as Framingham score determined by using the National Cholesterol Education Program (NCEP) calculator, available on-line at http://hp2010.nhlbihin.net/atpiii/calculator.asp

Written informed consent was obtained from all patients before the study. The study was approved by the hospital Ethics Committee and conforms to the ethical guidelines of the 1975 Declaration of Helsinki.

\section{Laboratory measurements}

Serum total cholesterol, HDL-cholesterol and triglycerides were measured by an Olympus AN 560 apparatus using an enzymatic colorimetric method. LDL-cholesterol levels were calculated according to the Friedwald formula. Plasma insulin levels were assayed by commercially available radioimmunoassay. The homeostasis model of insulin resistance (HOMA-IR), based on serum fasting glucose and insulin levels, was used as a measure of IR [26].

Adiponectin (APN) serum levels were measured with a commercial immunoassay (Tema Ricerca, Italy). Intra-assay and inter-assay coefficients of variation were 6 and 8\%, respectively. Serum levels of Cytokeratin 18-M30 were measured as marker of liver damage with a commercial immunoassay (Tema Ricerca, Italy) and expressed as mlU/ml. Intra-assay and inter-assay coefficients were $6 \%$ and $7 \%$ respectively.

\section{Ultrasonographic examination}

Liver US scanning was performed to assess the degree of steatosis. All US were performed by the same operator who was blinded to laboratory values using a GE Vivid S6 apparatus equipped with a convex 3,5 MHz probe. Liver steatosis was defined according to Hamaguchi criteria based on the presence of abnormally intense, high level echoes arising from the hepatic parenchyma, liverkidney difference in echo amplitude, echo penetration into deep portion of the liver and clarity of 
liver blood vessel structure [27,28]. Steatosis was assessed semi-quantitatively on a scale of 0-6: 0, absent; 1,2 mild; 3,4 moderate; 5,6 severe.

\section{Genetic analysis}

DNA was extracted from peripheral blood and purified by the The Wizard® Genomic DNA Purification Kit following the manufacturing protocol. Fluorogenic 5'-nucleotidase assays were developed to genotype the PNPLA3 rs $738409 \mathrm{C}$ to $\mathrm{G}$ nonsynonymous sequence variant, encoding I148M, in all subjects. The assay was performed using the TaqMan C7241_10 assay (Applied Biosystems, Foster City, CA) on ABI PRISM 7900 HT Sequence Detection Systems. (Applied Biosystems). The plate was run using standard condition at $95^{\circ} \mathrm{C}$ for $10 \mathrm{~min}, 95^{\circ} \mathrm{C}$ for $15 \mathrm{~s}$ then $60{ }^{\circ} \mathrm{C}$ for $1 \mathrm{~min}$ for 40 cycles. Allele frequencies were in Hardy-Weinberg equilibrium. The TaqMan assay was validated by direct sequencing of the SNP(rs738409) in representative samples of DNA on ABI PRISM 3130 XL Genetic Analyzer, and both methods gave identical results.

\section{Statistical analysis}

Statistical analysis was performed using the SPSS statistical software version $\underline{18.0}$ for Windows (SPSS, Inc., Chicago. Illinois). Student's t-test for unpaired data was used for the comparison of mean values. Distribution of continuous variables was tested for normality using the KolmogorovSmirnov test. Data are expressed as the mean \pm SD for normally distributed variables and as median and interquartile range for non-normally distributed data. Group comparisons were performed by use of analysis of variance (ANOVA) and unpaired Student's t-test when appropriate. Non normally distributed variables were tested by Mann-Whitney test and Kruskall-Wallis test. Proportions and categorical variables were tested by the $\chi^{2}$-test and by the 2-tailed Fisher's exact method when appropriate. All tests were two-tailed; a $P$ value of less than 0.05 was considered to indicate statistically significance. Multivariate analyses were performed using the stepwise logistic regression analysis testing for the dichotomous response variable presence or absence of MetS after 
controlling for possible clinical and biochemical confounders. The predictor variables entered in the different models were age, gender, PNPLA3-148MM, Hamaguchi score, HOMA-IR, serum lipid levels, serum ALT, adiponectin and cytokeratin-18 values, positive family history for diabetes and cardiovascular disease.

\section{Results}

Mean age was $54.5+11.9$ years. Among the 211 subjects with fatty liver, US examination revealed mild steatosis in 40, moderate steatosis in 90 and severe steatosis in 81 . Clinical and laboratory characteristics of subjects (mean age $54.5 \pm 11.9$ yrs) with different severity of fatty liver according to Hamaguchi's classification of steatosis are reported in Table 1. A progressive, statistically significant increase in the mean values of the indexes of central obesity (BMI, waist and hip circumferences), glucose metabolism (fasting blood glucose, insulin, HOMA-IR and glycosylated haemoglobin) serum alanine aminotransferase (ALT) and cytokeratine-18 was observed from the group with mild steatosis to the groups with moderate and severe steatosis; conversely, an opposite trend was observed for serum HDL cholesterol and adiponectin levels. No statistically significant between-group differences were observed for total and LDL cholesterol, triglycerides, aspartate aminotransferase (AST), $\gamma$-glutamyltransferase $(\gamma \mathrm{GT})$, smoking, and statin use.

The prevalence of MetS and its five clinical features defined according to ATPIII criteria is reported in Table 2. Prevalence of MetS progressively increased from subjects with mild steatosis to those with moderate and severe steatosis (from $52.5 \%$ to $65.2 \%$ and $82,3 \%$ respectively, $\mathrm{p}<0.01$ ). Compared to subjects with mild steatosis, the relative risk in those with severe steatosis was 1.3 (C.I. 1.0-1.6, $\mathrm{p}<0.02$ ) for increased waist circumference, 1.6 (C.I. 1.2-2.2, $\mathrm{p}<0.01$ ) for high blood pressure, 2.6 (C.I. 1.5-4.6, p<0.01) for high fasting glucose, 1.4 (C.I. 0.9-2.1 p=013) for high triglycerides and 1.9 (C.I. 1.0-3.4, p<0.05) for low HDL cholesterol. Clinical and biochemical variables of subjects with fatty liver according to the different PNPLA3 genotypes are reported in Table 3. The overall prevalence of PNPLA3-148II, PNPLA3-148IM and 
PNPLA3-148MM genotypes in our cohort was $45.0 \%(n=95), 40.7 \%(n=86)$ and $14.3 \%(n=30)$ respectively. A progressive, statistically significant decrease of mean serum triglycerides, Framingham cardiovascular risk score (from 9 (3/16.7) to 6 (2/13) and 4 (2/8.2) \% respectively, $\mathrm{p}<0.01$ ), MetS score and of the prevalence of MetS (from $71.6 \%$ to $72.1 \%$ and $50 \%$ respectively, $\mathrm{p}<0.05)$ was observed from the group with II genotype to those with genotypes IM and MM. The different groups were comparable with respect to age, BMI, waist circumference, US Hamaguchi score, serum total and LDL-cholesterol, GGT and cytokeratin-18.

Since at univariate analysis a lower prevalence of MetS was present in MM carriers, several models were generated by stepwise logistic analyses to assess the independent contribution of MM genotype for the prediction of MetS. In a stepwise logistic analysis including 10 clinical and biochemical covariates [Table 4], age, HOMA-IR, and the Hamaguchi score for steatosis were positive independent predictors of MetS, while a negative independent association was found between MetS and the homozygotes PNPLA3-I148M variant (overall predictive value of the model: $\left.\mathrm{R}^{2}=0.30\right)$.

\section{Discussion}

Our results show for the first time that PNPLA3-I148M gene variant is associated with a lower prevalence of MetS and reduced cardiometabolic risk in a large series of individuals with NAFLD. In fact, univariate and multivariate analyses suggest that individuals carrying the PNPLA3 MM genotype have a lower probability of having MetS.

Recently, the widely accepted assumption that NAFLD should be always considered the hepatic manifestation of MetS has been disputed and the study of PNPLA3-I148M polymorphism has been proposed as a useful tool to discriminate fatty liver with and without metabolic consequences and for estimating the risk for future cardiovascular or liver disease [18-19]. In fact, although the mechanisms underlining the association between PNPLA3 polymorphisms and NAFLD are still 
unclear, the I148M polymorphism seems to be associated to increased hepatic lipid accumulation and more severe histological liver disease progression [17,20-22].

Few studies have been performed to assess the different clinical phenotypes associated to PNPLA3 polymorphism and none referring to MetS as a clinical condition diagnosed according to standardized criteria; all refer only to some aspects of MetS; most contain small number of patients and report conflicting results.

In a recent study subjects homozygotes for the variant allele (PNPLA3-148MM) had significantly increased liver fat content with no insulin and triglyceride elevation, nor adipose tissue inflammation [16]. In a multi-ethnic study performed in Malaysia, where PNPLA3-I148M allele variation was associated with NASH susceptibility, NASH severity and the presence of fibrosis at liver histology, but not with simple steatosis and hypertriglyceridemia [17]. A further study performed in 335 individuals in Germany showed that PNPLA3-148MM genotype is strongly and independently associated with fatty liver but not with insulin resistance or estimates of liver injury [18]. In another study performed in Hispanic children and adolescents, liver fat in 148MM carriers was 2.7 times higher at nuclear resonance imaging than in wild type carriers and children with 148MM genotype had significantly lower HDL-cholesterol levels [19]. Moreover, a large study showed that variation at PNPLA3 confers a markedly increased risk of increasingly severe histological features of NAFLD, without a strong effect on some components of MetS [20]. Finally, a meta-analysis performed on 16 studies across different populations showed a strong influence of PNPLA3-I148M variation not only on liver fat accumulation, but also on the susceptibility of a more aggressive disease, with MM carriers having a greater risk of higher necro-inflammation and of developing fibrosis when compared with PNPLA3-I148I homozygotes [21].

By contrast, in a large study performed in a normoglycaemic population of Taiwan, PNPLA3148MM genotype was strongly associated with NAFLD and subjects had increased waist circumference and higher fasting insulin concentrations and higher HOMA-IR and ALT concentrations [29]. The reasons for these conflicting results are unclear and possibly depending on 
the ethnic differences on the relative role of insulin resistance and MetS in the pathogenesis of NAFLD [30].

In our study, NAFLD patients with PNPLA3-148MM genotype had a lower prevalence of MetS, which may suggest a lower global cardiovascular risk in PNPLA3-148MM carriers. Notably, low levels of serum triglycerides mainly accounted for the lower prevalence of MetS in PNPLA3MM subjects. These findings were also confirmed by the lower average calculated Framingham cardiovascular risk score in MM homozygotes as compared to wild type carriers.

Our results are consistent with the hypothesis that NAFLD might have two different clinical presentations, one associated to the common PNPLA3-I148I allele, presenting the clinical phenotype of MetS, insulin resistance and high cardiovascular risk, and the other, less frequent, associated to the PNPLA3-I148M allele, with a higher susceptibility to more severe liver histology and liver disease progression. Finally, our findings may also suggest that in the future genetic PNPLA3 screening could become an attractive novel target to identify individuals at high risk for cardio-metabolic diseases or for progressive liver disease.

The decreased triglyceridaemia found in our PNPLA3-148MM carriers is consistent with the results of a study showing a lower VLDL secretion in the liver of PNPLA3-148MM carriers, as compared to the PNPLA3-148II homozygotes, with PNPLA3-148MM genotype alone explaining 46\% of the variation in of VLDL triglyceride secretion. The Authors suggest that this might be the result of a reduced triglyceride hydrolysis in the adipose tissue leading to decreased VLDL synthesis and secretion and subsequent lipid deposition in the liver [31].

Our study may have some limitations. First, the study has been performed in patients recruited in a hospital-based setting. Second, fatty liver was assessed by US; the gold standard for the diagnosis of NAFLD is liver biopsy, but this is an invasive procedure with potentially serious complications and is therefore not acceptable without clinical indication. We acknowledge that grades of steatosis could have been better determined by magnetic resonance spectroscopy. However, Hamaguchi score showed $100 \%$ specificity and $91.7 \%$ sensitivity when compared with liver biopsy in NAFLD 
patients [26]. A further limitation is the higher prevalence of females in PNPLA3MM group which may have determined the lower cardiometabolic risk, considering the weight that gender has in the Framingham score. Finally, the rather low number of patients in the PNPLA3MM group may also represent a limitation to draw definitive conclusions based on genetic polymorphism.

\section{Conclusions}

We suggest that NAFLD may not always represent the hepatic manifestation of MetS. In fact, in our study, patients with NAFLD carrying PNPLA3-I148M gene had a lower prevalence of MetS and lower cardiometabolic risk. Further studies are needed to confirm the possible role of PNPLA3 polymorphisms to differentiate clinical phenotypes of NAFLD and to discriminate NAFLD with possible metabolic and cardiovascular consequences from fatty liver at risk for liver related complications.

Conflict of interests: All authors declare that they have no conflicts of interest.

\section{Learning points:}

- NAFLD does not always represent the hepatic manifestation of MetS

- PNPLA3 I148M gene variant is associated with a lower prevalence of metabolic syndrome and lower cardiometabolic risk. 


\section{References}

1. Ruhl CE, Everhart JE. Epidemiology of nonalcoholic fatty liver. Clin Liver Dis 2004; 8:501-19.

2. Bedogni G, Miglioli L, Masutti F, Tiribelli C, Marchesini G, Bellentani S. Prevalence of risk factors for non-alcoholic fatty liver disease: the Dionysos nutrition and liver study. Hepatology 2005; 42: 44-52

3. Neuschwander-Tetri BA, Caldwell SH. Nonalcoholic steatohepatitis: summary of an AASLD Single Topic Conference. Hepatology 2003; 37: 1202-19

4. Angulo P. Nonalcoholic fatty liver disease. N Engl J Med 2002; 346: 1221-31.

5. Day CP. Pathogenesis of steatohepatitis. Best Pract Res Clin Gastroenterol 2002; 16: 66378

6. Despres JP, Lemieux I, Bergeron J et al. Abdominal obesity and the metabolic syndrome: contribution to global cardiometabolic risk. Arterioscler Thromb Vasc Biol 2008; 28: 103949

7. Mottillo $\mathrm{S}$, Filion $\mathrm{KB}$, Genest $\mathrm{J}$ et al. The metabolic syndrome and cardiovascular risk: a systematic review and meta-analysis. J Am Coll Cardiol 2010; 56: 1113-32

8. Targher G, Day CP, Bonora E. Risk of cardiovascular disease in patients with nonalcoholic fatty liver disease. N Engl J Med 2010; 363: 1341-50

9. Del Ben M, Baratta F, Polimeni L, Angelico F. Non-alcoholic fatty liver disease and cardiovascular disease: epidemiological, clinical and pathophysiological evidences. Intern Emerg Med 2012; 7 (Suppl 3): S291-S296

10. Targher G, Marra F, Marchesini G. Increased risk of cardiovascular disease in nonalcoholic fatty liver disease: causal effect or epiphenomenon? Diabetologia 2008; 51:1947-53.

11. Lin YC, Lo HM, Chen JD. Sonographic fatty liver, overweight and ischemic heart disease. World J Gastroenterol 2005; 11:4838-42.

12. Mirbagheri SA, Rashidi A, Abdi S, Saedi D, Abouzari M. Liver: an alarm for the heart? Liver Int 2007; 27: 891-4.

13. Angelico F, Del Ben M, Conti R et al. Non alcoholic fatty liver disease: an hepatic consequence of common metabolic diseases. J Gastroenterol Hepatol 2003;18: 588-94.

14. Kotronen A, Yki-Järvinen H. Fatty liver: a novel component of the metabolic syndrome. Arterioscler Thromb Vasc Biol 2008; 28: 27-38.

15. Angelico F, Del Ben M, Conti R et al. Insulin resistance, the metabolic syndrome, and nonalcoholic fatty liver disease. J Clin Endocrinol Metab 2005; 90: 1578-82 
16. Lallukka S, Sevastianova K, Perttilä $\mathrm{J}$ et al. Adipose tissue is inflamed in NAFLD due to obesity but not in NAFLD due to genetic variation in PNPLA3. Diabetologia 2013; 56: 88692

17. Zain SM, Mohamed R, Mahadeva S et al. A multi-ethnic study of a PNPLA3 gene variant and its association with disease severity in non-alcoholic fatty liver disease. Hum Genet 2012; 131: 1145-52

18. Kantartzis K, Peter A, Machicao F et al. Dissociation between fatty liver and insulin resistance in humans carrying a variant of the patatin-like phospholipase 3 gene. Diabetes 2009; 58: 2616-2

19. Goran MI, Walker R, Le KA et al. Effects of PNPLA3 on liver fat and metabolic profile in Hispanic children and adolescents. Diabetes 2010; 59: 3127-3

20. Speliotes EK, Butler JL, Palmer CD et al. PNPLA3 variants specifically confer increased risk for histologic nonalcoholic fatty liver disease but not metabolic disease. Hepatology 2010; 52: 904-912

21. Sookoian S, Pirola CJ. Meta-analysis of the influence of I148M variant of patatin-like phospholipase domain containing 3 gene (PNPLA3) on the susceptibility and histological severity of nonalcoholic fatty liver disease. Hepatology 2011; 53: 1883-94

22. Dubuquoya C, Burnola AF, Moldesa M. PNPLA3, a genetic marker of progressive liver disease, still hiding its metabolic function? Clin Res Hepatol Gastroenterol 2012; 37: 30-5

23. Lallukka S, Sevastianova K, Perttila J, et al. Adipose tissue is inflamed in NAFLD due to obesity but not in NAFLD due to genetic variation in PNPLA3 Diabetologia 2013; 56: 88692.

24. American Diabetes Association. Diagnosis and classification of diabetes mellitus. Diabetes Care 2011; 34(Suppl. 1): S62-S69

25. Grundy SM, Cleeman JI, Daniels SR et al. Diagnosis and management of the metabolic syndrome: an American Heart Association/National Heart, Lung, and Blood Institute Scientific Statement. Circulation 2005; 112: 2735-52

26. Matthews DR, Hosker JP, Rudenski AS, Naylor BA, Treacher DF, Turner RC. Homeostasis model assessment: insulin resistance and beta-cell function from fasting plasma glucose and insulin concentrations in man. Diabetologia, 1985; 28: 412-19

27. Hamaguchi M, Kojima T, Itoh $\mathrm{Y}$ et al. The severity of ultrasonographic findings in nonalcoholic fatty liver disease reflects the metabolic syndrome and visceral fat accumulation. Am J Gastroenterol 2007; 102: 2708-15.

28. Saverymuttu SH, Joseph AEA, Maxwell JD. Ultrasound scanning in the detection of hepatic fibrosis and steatosis. Br Med J 1986; 292: 13-15

29. Wang CW, Lin HY, Shin SJ et al. The PNPLA3 I148M polymorphism is associated with insulin resistance and nonalcoholic fatty liver disease in a normoglycaemic population Liver Int 2011; 31: 1326-31 
30. Chang CY. Understanding the relationship between PNPLA3, NAFLD and insulin resistance: do ethnic differences bring more questions or more answers? Liver Int 2011; 31: 1246-1249

31. Pirazzi C, Adiels M, Burza MA et al. Patatin-like phospholipase domain-containing 3 (PNPLA3) I148M (rs738409) affects hepatic VLDL secretion in humans and in vitro. J Hepatol 2012; 57: 1276-82 
Table 1. Clinical and biochemical characteristics of subjects according to severity of nonalcoholic fatty liver disease (NAFLD) at ultrasonography

\begin{tabular}{|c|c|c|c|c|}
\hline & \multicolumn{3}{|c|}{ NAFLD } & \multirow[b]{2}{*}{$\mathbf{p}$} \\
\hline & $\begin{array}{c}\text { Mild } \\
(n=40)\end{array}$ & $\begin{array}{c}\text { Moderate } \\
\quad(n=90)\end{array}$ & $\begin{array}{c}\text { Severe } \\
(n=81)\end{array}$ & \\
\hline $\begin{array}{l}\text { Gender }(\mathbf{F} / \mathbf{M}) \% \\
\text { Age }(\mathrm{yrs}) \\
\text { BMI }(\mathrm{kg} / \mathrm{m} 2) \\
\text { Waist circumference }(\mathrm{cm})\end{array}$ & $\begin{array}{c}45.0 / 55.0 \\
49.9(14.6) \\
29.6(5.1) \\
103,5(97,3 / 109,5)\end{array}$ & $\begin{array}{c}36.7 / 63.3 \\
56.0(11.5) \\
30.3(5.3) \\
105(98 / 113)\end{array}$ & $\begin{array}{c}29.6 / 70.4 \\
54.6(10.4) \\
33.5(5.3) \\
113(106 / 125)\end{array}$ & $\begin{array}{l}\mathrm{ns} \\
\mathrm{ns} \\
<.001 \\
<.001\end{array}$ \\
\hline $\begin{array}{l}\text { Fasting plasma glucose }(\mathrm{mg} / \mathrm{dl}) \\
\text { Insulin }(\mathrm{mU} / \mathrm{l}) \\
\text { HbA1c }(\%) \\
\text { HOMA IR } \\
\text { Total Cholesterol }(\mathrm{mg} / \mathrm{dl}) \\
\text { Triglycerides }(\mathrm{mg} / \mathrm{dl}) \\
\text { HDL cholesterol }(\mathrm{mg} / \mathrm{dl}) \\
\text { LDL cholesterol }(\mathrm{mg} / \mathrm{dl}) \\
\text { AST }(\mathrm{UI} / \mathrm{l}) \\
\text { ALT }(\mathrm{UI} / \mathrm{l}) \\
\gamma \text { GT }(\mathrm{UI} / \mathrm{l}) \\
\text { Cytokeratin } 18(\mathrm{mIU} / \mathrm{ml}) \\
\text { Adiponectin }(\mathrm{ng} / \mathrm{ml}) \\
\text { Framingham cardiovascular } \\
\text { risk score }(\%)^{*}\end{array}$ & $\begin{array}{c}92(86 / 100,5) \\
11,5(7,7 / 15,5) \\
5,4(5,1 / 5,7) \\
2.5(1.6 / 3.7) \\
209(41) \\
132,5(188,3 / 175 / 8) \\
53(43 / 60) \\
125(34) \\
19(16 / 26,8) \\
21(17,3 / 34) \\
23(16,5 / 42,3) \\
169,5(129,3 / 183,8) \\
10,3(5,5 / 12) \\
5(1 / 14,5)\end{array}$ & $\begin{array}{c}97(92,8 / 117,5) \\
13(9,3 / 17,9) \\
5,6(5,3 / 6,1) \\
3.1(2.2 / 4.7) \\
201(40) \\
138(99,8 / 179) \\
45,5(39,8 / 60,3) \\
121(35) \\
21(18 / 25) \\
26(20,8 / 40) \\
21(16 / 36) \\
176(140 / 190) \\
8,8(6 / 13,1) \\
6(3 / 13)\end{array}$ & $\begin{array}{c}107(95 / 128,3) \\
16,6(12,5 / 24,5) \\
6(5,6 / 6,5) \\
5.2(3.1 / 7.3) \\
198(40) \\
152,5(119,5 / 197,8) \\
43(38 / 51) \\
118(33) \\
22(18 / 32) \\
34(24 / 45) \\
32(23,8 / 55) \\
180(169,5 / 192,5) \\
6,0(4,9 / 9,1) \\
3(9 / 14)\end{array}$ & $\begin{array}{l}<.001 \\
<.001 \\
<.001 \\
<.001 \\
\mathrm{~ns} \\
\mathrm{~ns} \\
<.05 \\
\mathrm{~ns} \\
\mathrm{~ns} \\
<.05 \\
\mathrm{~ns} \\
<.01 \\
<.001 \\
\mathrm{~ns}\end{array}$ \\
\hline *see Methods & & & & \\
\hline
\end{tabular}


Table 2. Prevalence of MetS and its clinical features in subjects with different severity of steatosis at liver ultrasonography.

\begin{tabular}{l|c|c|c|c}
\hline & \multicolumn{3}{|c|}{ NAFLD } & \\
\cline { 2 - 5 } & $\begin{array}{c}\text { Mild } \\
(\mathrm{n}=40)\end{array}$ & $\begin{array}{c}\text { Moderate } \\
(\mathrm{n}=90)\end{array}$ & $\begin{array}{c}\text { Severe } \\
(\mathrm{n}=81)\end{array}$ & $\mathbf{p}$ \\
\hline Metabolic Syndrome (\%)* & 52.5 & 65.2 & 82.3 & $<.01$ \\
High blood pressure (\%)* & 55 & 78.9 & 88.6 & $<.001$ \\
High waist circumference (\%)* & 70 & 75 & 91.1 & $<.01$ \\
High fasting blood glucose (\%)* & 25 & 44.4 & 65 & $<.001$ \\
High triglycerides (\%)* & 40 & 44.4 & 55 & $\mathrm{~ns}$ \\
Low HDL-cholesterol (\%)* & 25 & 35.6 & 46.3 & $\mathrm{~ns}$ \\
MetS Score** & $3(2 / 4)$ & $3(2 / 4)$ & $4(3 / 5)$ & $<.01$ \\
\hline
\end{tabular}

*Defined according to ATPIII criteria; **see Methods 
Table 3. Clinical and biochemical characteristics of subjects with different PNPLA3 genotypes

\begin{tabular}{|c|c|c|c|c|}
\hline \multicolumn{5}{|c|}{ PNPLA3 genotypes } \\
\hline & $\begin{array}{l}\text { 148II } \\
(95)\end{array}$ & $\begin{array}{l}\text { 148IM } \\
(86)\end{array}$ & $\begin{array}{l}\text { 148MM } \\
\text { (30) }\end{array}$ & $\mathbf{p}$ \\
\hline Gender (F/M) \% & $27.4 / 72.6$ & $39.5 / 60.5$ & $50 / 50$ & $<.05^{*}$ \\
\hline Metabolic Syndrome (\%) & 71.6 & 72.1 & 50 & $<.05 *$ \\
\hline Age (yrs) & $54 \pm 11$ & $54 \pm 12.8$ & $54.3 \pm 12.1$ & ns \\
\hline Weight $(\mathrm{kg})$ & $92.2 \pm 18.1$ & $89.0 \pm 16.5$ & $89.8 \pm 16.2$ & ns \\
\hline BMI $(\mathrm{kg} / \mathrm{m} 2)$ & $32.1 \pm 5.6$ & $30.4 \pm 5.1$ & $31.7 \pm 6.1$ & ns \\
\hline Waist circumference $(\mathrm{cm})$ & $110(103.5 / 118)$ & $105(101 / 113.5)$ & $106(96.7 / 118.5)$ & ns \\
\hline Fasting plasma glucose $(\mathrm{mg} / \mathrm{dl})$ & $101(92 / 110)$ & $97(92 / 121)$ & $101(87.5 / 128.2)$ & ns \\
\hline Insulin $(\mu \mathrm{U} / \mathrm{ml})$ & $14.1(10.7 / 20.2)$ & $13.7(10.1 / 19.4)$ & $11.6(8.7 / 16.8)$ & ns \\
\hline HOMA IR & $3.6(2.3 / 5.8)$ & $3.5(2.5 / 5.7)$ & $2.8(2.0 / 5.6)$ & ns \\
\hline Total Cholesterol (mg/dl) & $205 \pm 41.9$ & $198 \pm 40.3$ & $196.9 \pm 34.3$ & $\mathrm{~ns}$ \\
\hline Triglycerides (mg/dl) & $157(112 / 193)$ & 141(107.7/185) & 111.5(90.7/148.7) & $<.01 *$ \\
\hline HDL cholesterol (mg/dl) & $45(39 / 53)$ & $44(38 / 56)$ & $50.5(43 / 61)$ & $\mathrm{ns}$ \\
\hline AST (UI/l) & $20(17 / 25)$ & $21(18 / 31)$ & $23(17.7 / 27.7)$ & $\mathrm{ns}$ \\
\hline ALT (UI/l) & $26(19 / 35)$ & $30.5(22 / 45)$ & $30(22.7 / 40.5)$ & ns \\
\hline$\gamma \mathbf{G T}(\mathrm{UI} / \mathrm{l})$ & $28(19 / 42)$ & $28(17 / 46)$ & $22(17.5 / 39.5)$ & $\mathrm{ns}$ \\
\hline Cytokeratine $18(\mathrm{mIU} / \mathrm{ml})$ & $180(143 / 192)$ & $180(151.2 / 190)$ & $170.5(130 / 182.2)$ & ns \\
\hline Adiponectin (ng/ml) & $7.5(5 / 11)$ & $7.5(5.5 / 12)$ & $9.3(4.9 / 13.1)$ & ns \\
\hline $\begin{array}{l}\text { Framingham cardiovascular } \\
\text { risk score }(\%)\end{array}$ & $9(3 / 16.7)$ & $6(2 / 13)$ & $4(2 / 8.2)$ & $<.01 *$ \\
\hline Hamaguchi score & $4(3 / 5)$ & $4(3 / 5)$ & $4(3 / 5)$ & $\mathrm{ns}$ \\
\hline MetS score ${ }^{* *}$ & $4(2 / 5)$ & $3(2 / 4)$ & $2(1,75 / 4)$ & $<.01 *$ \\
\hline
\end{tabular}

*148MM vs 148II; **see Methods 
Table 4. Multiple logistic analysis of independent predictors of metabolic syndrome in 211 subjects with non-alcoholic fatty liver disease.

\begin{tabular}{lcccccc} 
& B & S.E. & P. & \multicolumn{2}{c}{ O.R. } & \multicolumn{2}{c}{$\mathbf{9 5 , 0 \%}$ C.I. for O.R. } \\
\cline { 2 - 7 } Age & & & & & Lower & Upper \\
PNPLA3.148MM & .062 & .015 & .000 & 1.064 & 1.032 & 1.096 \\
HOMA-IR & -1.195 & .460 & .009 & .303 & .123 & .746 \\
Constant & .267 & .083 & .001 & 1.306 & 1.110 & 1.538 \\
& -3.234 & .858 & .000 & .039 & &
\end{tabular}

Variables entered on step 1: age, gender, PNPLA3.148MM, Hamaguchi score, HOMA-IR, serum ALT serum adiponectin, positive family history for diabetes and cardiovascular disease. 


\section{Highlights:}

1. Non-alcoholic fatty liver disease (NAFLD) is usually considered the hepatic consequence of the metabolic syndrome.

2. Polymorphisms of patatin-like phospholipase domain-containing protein 3 (PNPLA3) gene may predispose to NAFLD and its progression.

3. We evaluated whether PNPLA3 gene variants may predict different clinical phenotypes of NAFLD patients.

4. Patients with PNPLA3 I148M gene variant have lower prevalence of metabolic syndrome and lower cardiometabolic risk. 